\title{
Evaluation of Efficacy of Ultrasound Guided Paravertebral Nerve Block as Postoperative Analgesia in Abdominal Surgeries
}

\author{
Samia Abd El Mohsen Abd El Latief, Sanaa Mohamed El Fawal, Sabah Naguib Barsom, Mohamed \\ Mohsen Mohamed Ali
}

\begin{abstract}
Anesthesiology and Intensive Care Medicine Department, Faculty of Medicine, Ain Shams University
Corresponding Author: Mohamed Mohsen Mohamed Ali, E-mail: mohamed.mohsen.habashi@ gmail.com, Phone: 01026626226
\end{abstract}

\begin{abstract}
Background: abdominal surgeries requires either general or regional anesthesia. Normally, general anesthesia needed for abdominal surgeries consists of a mixture of neuromuscular blockers, opioids and inhalation anesthetics. Airway control can be achieved by either endotracheal intubation or laryngeal mask. Regional anesthesia can be used alone in abdominal surgeries or in combination with general anesthesia. If regional anesthesia is used alone then effective sedation is required. Pulmonary functions impairment has a higher incidence of happening in upper abdominal surgeries than non-abdominal and non-thoracic surgeries. Intraoperative patient's ventilation is closely related to the amount of the analgesics received give that depression of ventilation and excessive sedation should be avoided. The postoperative length of hospital stay prolongs after major gastro-intestinal surgeries due to immobility, pain and gastric immobility and that's why postoperative pain control is essential to speed up mobility and gastro-intestinal functions.

Objectives: the aim of this study was to evaluate the efficacy of ultrasound-guided paravertebral nerve block as postoperative analgesia in abdominal surgeries.

Patients and Methods: a total of 64 patients were assessed for eligibility to our study, but two patients not meeting inclusion criteria and other two patients were declined to participate so four patients were excluded from the study. Sixty ASA physical status I and II patients of both sex, 18-60 years old, undergoing abdominal surgery in surgical operating theater of El-Sheikh Zayed Specialized hospital and Ain-Shams Surgery hospital, who fulfilled the inclusion criteria, had been enrolled in the study. All enrolled patients have already completed the study. Those patients were randomly allocated into two groups, 30 patients each.

Results: demographic data including (age, gender and type of operation) did not show any statistically significant difference between the two studied groups with $\mathrm{P}=0.176$ for the age and $\mathrm{P}=1.0$ for the gend er and the type of operation. Patients' characteristics included age, sex, ASA score and type of operation. There was no significant difference in the demographic data of the two groups of the study. The mean age was 49.9 year in the Paravertebral block group, while the mean age was 47.6 year in the control group. The females' count in group A and group B exceed the males' amount with $20 \%$ and $6.66 \%$ respectively. According to the ASA classification, both groups have $60 \%$ classified as ASA-1 and $40 \%$ as ASA-2. In this study, four types of operations are done, Lap-cholecystectomy, Umbilical Hernia, Appendectomy and Inguinal Hernia.

Conclusion: this study proved that PVB provided better postoperative pain control, fewer opioids consumption, less postoperative nausea and vomiting compared with other analgesic treatment strategies, indicating that a postoperative PVB is a feasible and an effective method for an improved postoperative pain treatment after abdominal surgery. The current study concluded that there was a significant difference in post-operative period regarding morphine consumption between PVB group and control group with the mean of total morphine consumption 0.9 and 6.9 respectively, with the $\mathrm{P}$-value $<0.001$.
\end{abstract}

Keywords: Ultrasound Guided Paravertebral Nerve Block, Postoperative Analgesia, Abdominal Surgeries

\section{INTRODUCTION}

Abdominal surgeries requires either general or regional anesthesia. Normally, general anesthesia needed for abdominal surgeries consists of a mixture of neuromuscular blockers, opioids and inhalation anesthetics. Airway control can be achieved by either endotracheal intubation or laryngeal mask. Regional anesthesia can be used alone in abdominal surgeries or in combination with general anesthesia. If regional anesthesia is used alone then effective sedation is required ${ }^{(\mathbf{1})}$.
Pulmonary functions impairment has a higher incidence of happening in upper abdominal surgeries than non-abdominal and non-thoracic surgeries. Intraoperative patient's ventilation is closely related to the amount of the analgesics received give that depression of ventilation and excessive sedation should be avoided. The postoperative length of hospital stay prolongs after major gastro-intestinal surgeries due to immobility, pain and gastric immobility and that's why postoperative pain control is essential to speed up mobility and gastro-intestinal functions ${ }^{(2)}$. 
Thoracic paravertebral block (PVB) has been demonstrated to provide effective postoperative analgesia in patients undergoing minor and major abdominal surgery by blocking sensory innervation of the abdominal wall. PVB significantly reduces the need for supplemental opioid administration, reduces postoperative nausea and vomiting (PONV), and in some patient groups shortens hospital stay. Although PVB has been demonstrated to be effective in patients undergoing abdominal and thoracic surgery, its analgesic efficacy after major gynecological surgery has not been reported yet ${ }^{(3)}$.

Paravertebral nerve block was a popular technique in the early 20th century. However, for some reason, paravertebral nerve block lost popularity and was almost extinct until the late 1970s, when there was a renewed interest in the technique. Recently, this technique was reviewed and found to be safe and efficacious. A paravertebral block is essentially a unilateral block of the spinal nerve, including the dorsal and ventral rami, as well as the sympathetic chain ganglion. These blocks can be performed at any vertebral level. However, they are most commonly performed at the thoracic level because of anatomic considerations ${ }^{(4)}$.

Thoracic paravertebral block (TPVB) is the technique of injecting local anesthetic adjacent to the thoracic vertebra close to where the spinal nerves emerge from the intervertebral foramina. This result in ipsilateral somatic and sympathetic nerve blockade in multiple contiguous thoracic dermatomes above and below the site of injection.It is effective in treating acute and chronic pain of unilateral origin from the chest and abdomen. Bilateral use of TPVB has also been described. Our understanding of the safety and efficacy of TPVB has improved significantly in the last two decades, prompting its use in children and neonates and for surgical anesthesia ${ }^{(5)}$.

\section{AIM OF THE WORK}

The aim of this study is to evaluate the efficacy of ultrasound-guided Paravertebral nerve block as postoperative analgesia in abdominal surgeries.

\section{PATIENTS AND METHODS}

Study Design: Sixty patients are scheduled for abdominal surgery, are randomly divided into two equal groups. Each group consists of 30 patients $(n=30)$.
Group A: This group will receive general anesthesia, after completion of surgery and before extubation, paravertebral block will be performed by ultrasound using L-bupivacaine as postoperative analgesia.

Group B: This group will serve as the control group, members of this group received general anesthesia only. Postoperative analgesia in the form of NSAIDs and Opioids will be given, if needed.

Inclusion Criteria: Patient's approval. American Society of Anesthesiologists (ASA) physical status I, II. Age between 18-60. Patients undergoing abdominal surgery.

Exclusion Criteria: Patient's refusal. American Society of Anesthesiologists (ASA) physical status III, IV, V and VI. Sensitivity to local anesthetics. Local sepsis. Tolerance to opioids. Coagulopathy. Chest Disease. Anomalies of spine.

Methods: This study was carried out at ElSheikh Zayed Specialized Hospital and Surgery Hospital of Ain-Shams University. The Anesthesiology and Intensive Care Medicine Department of Ain-Shams University approved the study protocol. All patients were informed about the study design and objectives as well as tools and techniques. Every patient prior to the study signed informed consent. Patients were randomly allocated to one of two groups by sealed closed envelope technique. Surgeries included Appendectomy, Umbilical and Inguinal Hernia Repair and Laparoscopic cholecystectomy. The study was approved by the Ethics Board of Ain Shams University and an informed written consent was taken from each participant in the study.

Group A (Paravertebral Block Group): Patients $(n=30)$ of Group A will receive in plane paramedian sagittal paravertebral block (PVB) by two injections at two levels from $T_{7}$ to $L_{1}$ space unilateral according to type of surgery(e.g. Appendectomy $\mathrm{T}_{10}$ \& $\mathrm{T}_{11}$, Laparoscopic Cholecystectomy $\mathrm{T}_{6^{-}} \mathrm{T}_{7}$, Inguinal Hernia Repair $\mathrm{T}_{9}-\mathrm{T}_{11}$, Umbilical Hernia Repair $\mathrm{T}_{12}-\mathrm{L}_{1}$ ). The scanning probe is the linear multi-frequency 13-16 MHz Patients of Group A will receive the block after the completion of the surgery and right before the extubation.

Group B (General Anesthesia only group): Patients $(n=30)$ of this group will receive General Anesthesia only. After completion of surgery and after extubation, Group B will also receive the postoperative analgesia regimen including NSAIDs and opioids when needed. The amount of NSAIDs and opioids given to 
the patients of Group B will be recorded along with the percentage of incidence of postoperative nausea and vomiting also with calculation of Hospital stay and early mobilization of patients.

Monitoring and Data Collection: The below data was collected and monitored postoperative for both groups.

\section{Pain score.}

Hemodynamic.

\section{Postoperative nausea and vomiting:}

Nausea and vomiting were managed by IV injection of $10 \mathrm{mg}$ metoclopramide.

\section{Rescue analgesia.}

\section{Statistical Analysis}

Data was analyzed using IBM SPSS advanced statistics (Statistical Package for Social Sciences), version 21 (SPSS Inc., Chicago, IL). Numerical data was described as mean and standard deviation, median and range as appropriate, while qualitative data was described as number and percentage.

\section{RESULTS}

Table (1): Age in both study groups

\begin{tabular}{|l|c|c|c|c|c|}
\hline & \multicolumn{2}{|c|}{ Group A } & \multicolumn{2}{c|}{ Group B } & \multirow{2}{*}{ P-value } \\
\cline { 2 - 5 } & Mean & SD & Mean & SD & \\
\hline Age (years) & 49.9 & 4.0 & 47.6 & 6.3 & $\mathbf{0 . 1 7 6}$ \\
\hline
\end{tabular}

Comparison between group A and group B regarding age (years).

Table (2): Sex distribution, ASA Score and Type of Operation in both groups

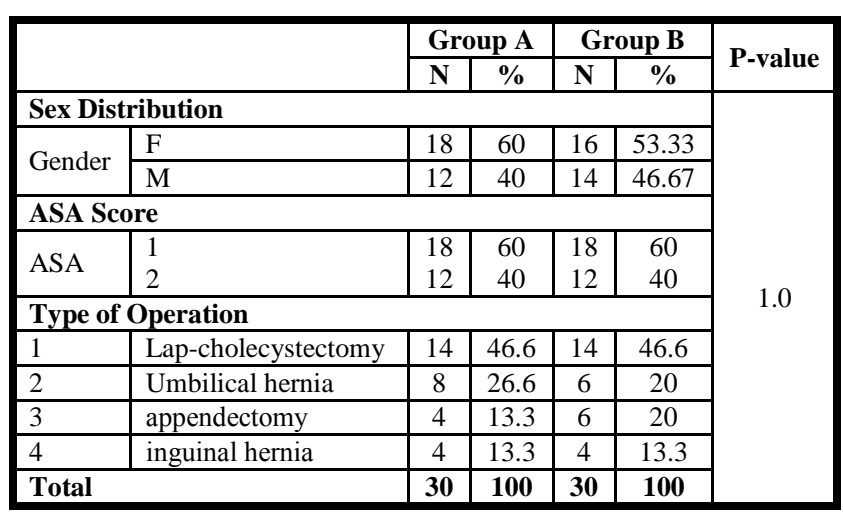

$* \mathrm{P}$-value is significant with significance level $<=1$

Comparison between group A and group B regarding sex distribution, ASA score, and type of operation.
Table (3): Median, range, mean and SD of VAS Score in both groups

\begin{tabular}{|l|c|c|c|c|c|c|c|}
\hline \multirow{2}{*}{ Characteristics } & \multicolumn{3}{|c|}{ Group A } & \multicolumn{3}{c|}{ Group B } & \multirow{2}{*}{ P-value* } \\
\cline { 2 - 8 } & $\begin{array}{l}\text { Median } \\
\text { (range) }\end{array}$ & Mean & SD & $\begin{array}{l}\text { Median } \\
\text { (range) }\end{array}$ & Mean & SD & \\
\hline VAS 0h & $1(0-2)$ & 1 & 0.6124 & $6(3-7)$ & 5.5 & 1.118 & $\mathbf{0 . 0 0 1}$ \\
\hline VAS 0.5 h & $1(0-3)$ & 1.25 & 0.9014 & $4(3-6)$ & 4.25 & 0.9014 & $\mathbf{0 . 0 0 1}$ \\
\hline VAS 1 h & $2(0-6)$ & 2.5 & 1.7321 & $3(2-6)$ & 3.5 & 1.1547 & $\mathbf{0 . 0 3}$ \\
\hline VAS 3 h & $2(0-4)$ & 2 & 1.1726 & $3(2-4)$ & 3 & 0.6124 & $\mathbf{0 . 0 0 1}$ \\
\hline VAS 6 h & $1(0-3)$ & 1.25 & 0.9014 & $3(2-6)$ & 3.5 & 1.1547 & $\mathbf{0 . 0 0 1}$ \\
\hline VAS 12 h & $1(0-6)$ & 2 & 1.7678 & $3(2-6)$ & 3.5 & 1.1547 & $\mathbf{0 . 0 0 1}$ \\
\hline VAS 24 h & $1(0-3)$ & 1.25 & 0.9014 & $3(2-3)$ & 2.75 & 0.1443 & $\mathbf{0 . 0 0 1}$ \\
\hline
\end{tabular}

$* \mathrm{P}$-value is significant with significance level $<=0.05$.

Comparison between group A and group B regarding VAS $0 \mathrm{~h}$, VAS $0.5 \mathrm{~h}$, VAS $1 \mathrm{~h}$, VAS $3 \mathrm{~h}$, VAS 6 h, VAS 12 h, VAS 24 h.

\section{DISCUSSION}

In a study conducted by Agarwal et al. ${ }^{(6)}$ patients were included in prospective randomized study and allocated to two groups: Group A (25 patients) receiving general anesthesia alone and Group B (25 patients) receiving nerve-stimulatorguided bilateral thoracic Paravertebral Block (PVB) at $\mathrm{T}_{7}$ level with $0.3 \mathrm{ml} / \mathrm{kg}$ of $0.25 \%$ bupivacaine prior to induction of general anesthesia. Intraoperative analgesia was supplemented with fentanyl $(0.5 \mu / \mathrm{kg})$ based on hemodynamic and clinical parameters. Postoperatively, patients in both the groups received Patient-Controlled Analgesia (PCA) morphine for the first 24 hours. The efficacy of PVB was assessed by comparing intraoperative fentanyl requirements, postoperative VAS scores at rest, and on coughing and PCA morphine consumption between the two groups. Agarwal ${ }^{(6)}$ study pre-induction PVB resulted in improved analgesia for 24 hours following laparoscopic cholecystectomy in this study, along with a significant reduction in perioperative opioid consumption and opioid-related side effects which strongly agrees with the current study which concluded that there was a significant difference in post-operative period regarding morphine consumption between PVB group and control group with the mean of total morphine consumption 0.9 and 6.9 respectively, with the $\mathrm{P}$-value $<0.001$.

Agarawel (6) study PCA morphine requirement was significantly low in the PVB group at 2, 6, 12, and 24 hours postoperatively compared to that in Group A (4.4 mg vs. $6.9 \mathrm{mg}$, 
$7.6 \mathrm{mg}$ vs. $14.2 \mathrm{mg}, 11.6 \mathrm{mg}$ vs. $20.0 \mathrm{mg}, 16.8 \mathrm{mg}$ vs. $27.2 \mathrm{mg}$, respectively; $\mathrm{P}<0.0001$ at all intervals). Despite that the current study measured total morphine consumption postoperatively and Agarawel's study did that periodically however, both studies show high efficacy of PVB in postoperative analgesia.

In a study conducted by Fentie et al. ${ }^{(7)}$, Fifty-two patients were prospectively enrolled. Of those 52 patients, 50 were eligible to complete the study while 2 patients were eliminated because of not matching the study criteria. The patients were divided in two equal groups. The two groups are the control group and the group that received the paravertebral block. Pain intensity will be measured numeric pain rating scale along with the first analgesic administration and opioid consumption will be measured during the first 24 hours after the operation. the paravertebral block group received $15-20 \mathrm{ml}$ bupivacaine $0.5 \%$ while the other group had adequate opioid analgesics. The difference between Fentie's study and the current study that the current study used VAS (Visual Analog Score) pain scoring system in of NRS (Numerical rating scale) pain scoring system. Twenty-four hours after surgery, NRS at rest was 4 (3-6) vs. 5 (5-7) and at movement 4 (4-7) vs. 6 (5-7.5) for the PVB and control groups, respectively. The difference between the groups over the whole observation period was statistically significant $(P<0.05)$. In the current study, group $\mathrm{A}$ (PVB group) scored VAS score twenty-four hours after surgery 1 (0-3) with a mean of 1.25 while Group B (without PVB) scored 3 (2-3) with a mean of 2.75. In Fentie's study Twenty-four hours after surgery, median (25th-75th percentile) total morphine consumption was $0(0-2)$ vs. $2.5(2-4)$ $\mathrm{mg}(P<0.0001)$ while in the current study total morphine consumption was $0.9 \mathrm{mg}$ by group $\mathrm{A}$ (PVB group) vs. $6.9 \mathrm{mg}$ by group B (without PVB).After surgery, Fentie's study along with the current study showed that the mean first analgesic requirement time was prolonged in the PVB group in statistically significant fashion $(P<0.001){ }^{(7)}$.

The previously mentioned study strongly supports the results of the current study stating that Single-shot thoracic PVB as a component of multimodal analgesic regimen provided superior analgesia when compared with the control group up to 24 postoperative hours after cholecystectomy.
In a study conducted by Ben-David et al. (8), 100 patients had a radical prostatectomy surgery. They were examined after the surgery knowing that they had the same surgeon. In 50 patients, ketorolac $30 \mathrm{mg}$ iv was administered along with $30 \mathrm{ml}$ bupivacaine $0.25 \%$ wound infiltration at wound closure. After the operation the patients received $15 \mathrm{mg}$ IV ketorolac every 6 hours for 2 days and a PCA device was administered containing opioids. The other 50 patients, in addition to the previously mentioned medications, received before the surgery one oral dose of Valdecoxib $40 \mathrm{mg}$ and bilateral paravertebral injection of Ropivicaine $0.5 \%$ at $\mathrm{T}_{10}$ $\mathrm{T}_{11} \mathrm{~T}_{12}(5 \mathrm{ml}$ in each level). Quantities of opioids used, pain scores and hospital length of stay were all recorded.

The previously mentioned study supports the results of the current study according to the immediate postoperative reduction of pain score. On the contrary, the current study has a higher incidence of Post-Operative Nausea \& Vomiting in Group B (without PVB) in comparison with Group A (with $\mathrm{PVB}$ ), with $\mathrm{P}$ value $<0.001$ than the previously mentioned study. In a study conducted by Naja et al. (9), sixty patients which were prospectively allocated to receive either bilateral paravertebral nerve blockade or general anesthesia for ventral hernia repair. The end-points of the study were length of hospital stay, postoperative analgesia (visual analogue scale, supplemental opioid requirement) and incidence of postoperative nausea and vomiting. In agreement with the current study and although it used the Paravertebral block postoperatively however Naja's study found out that Bilateral paravertebral blockade combined with light intravenous sedation was superior to general anesthesia for ventral hernia repair. Paravertebral blockade was associated with shorter hospital stay, improved analgesia and less postoperative nausea and vomiting. It is suggested that this technique deserves more widespread use in patients undergoing ventral hernia repair. In 2008, study on 24 adult patients, aged 18-50 years, ASA-I-II, of both sexes scheduled for right lobe donor hepatic resection, were enrolled in this study. Patients were randomly allocated into 2 equal groups of 12 patients each. Before induction of general anesthesia, all patients received a bilateral single-site thoracic paravertebral injection at the level of $\mathrm{T}_{7}-\mathrm{T}_{8}$ in the sitting position. Patients of Group B were 
injected with $25 \mathrm{~mL}$ of bupivacaine $0.25 \%$ with epinephrine 1:400.000 on each side. Patients of Group P were injected with $25 \mathrm{~mL}$ of $0.9 \% \mathrm{NaCl}$ (placebo). General anesthesia was standardized in all patients. Postoperative pain score, analgesic requirements and the incidence of postoperative nausea and vomiting were recorded ${ }^{(9)}$.

Although the current study didn't use placebo injection in Group B (without PVB) however the results of Moussa's study enrolled patients with the same ASA scoring as the current study. Both studies also assessed postoperative pain score, analgesic requirements and incidence of postoperative nausea and vomiting and both studies concluded that single-site thoracic paravertebral block is an easy, safe and efficient technique for postoperative pain management in patients undergoing abdominal surgeries. In a study conducted by Jin et al. ${ }^{(10)}$, a total of 72 patients underwent breast cancer surgery. Patients were divided in two groups randomly and equally. Each group received thoracic Paravertebral block by $20 \mathrm{ml}$ of bupivacaine $0.25 \%$ then vital signs including Systolic Blood Pressure (SBP), Diastolic Blood Pressure (DBP) and pulse (HR) were recorded. The Visual Analogue Scale (VAS) scoring system measured the postoperative pain intensity. Amount of opioid and non-opioid analgesics administered were recorded. Significant differences were observed in $\mathrm{HR}(P<.05)$, SBP $(P<.05)$, and DBP $(P<.05)$ at the 30-minute point during surgery between the 2 groups. In addition, the time of the first administration of analgesia $(P=.043)$ and the mean consumption of analgesic agents $(P=.035)$ in the intervention group were much better than those in the control group. However, no significant differences in HR or VAS were found at any time point after surgery $(P>.05)$. Furthermore, similar adverse events were detected in both groups $(P>.05)^{(\mathbf{1 0})}$.

Although the previously mentioned study didn't find any statistical significance in HR and VAS scoring $(P>.05)$ however, it supports the result of the current study according to the effect of PVB on postoperative vital signs including systolic and diastolic blood pressure in addition to the mean consumption of analgesics and time elapsed before first administration of analgesia. A study made by Kehlet \& Dahl showed that not only the pain is superior in TPVB group but has a positive effect on immune system. Postoperative nausea and vomiting (PONV) are being the common irritating complains to any patient especially during the first 24 hours following surgery. Since Opioids are given intravenously proved to have several disadvantages, including PONV and a delay in the recovery of body functions such as bowel movement and mobilization, several studies have focused on the use of regional analgesia to overcome this complaint postoperatively ${ }^{(11)}$.

Data represented in this study showed that postoperative complications, nausea, and vomiting, which resulted due to systemic use of opioids were mostly among the control group rather than the TPVB group $(\mathrm{P}<0.001)$. The reason for this could be that the requirement of morphine among the control group was higher than the other group. Paravertebral block has fewer side effects such as urinary retention or hypotension compared to epidural anesthesia but these side effects were not recorded in this study. In this study there was a statistical significant difference in morphine consumption postoperatively between control group and TPV block group. The mean morphine consumption was $0.9 \mathrm{mg}$ in the paravertebral group while in control group was 6.9 $\mathrm{mg}(\mathrm{p}<0.001)$.The current study showed that there was no incidence of complications related to the technique, however, it is mainly due to direct visualization of paravertebral space and real-time injection of the local anesthetic under ultrasound guidance even though due to negative aspiration before injection. Other disadvantages of this technique include difficulty of maneuver if patient is awake. Also, requirement of more preoperative time for application of the block and incidence of vascular puncture hypotension hematoma signs of epidural or intrathecal spread plural puncture or pneumothorax. Further studies are highly recommended to insert a catheter in thoracic paravertebral space. Join studies with the surgical team to evaluate the long-term outcomes of chronic pain are also recommended ${ }^{(\mathbf{1 2})}$.

\section{CONCLUSION}

This study proved that PVB provided better postoperative pain control, fewer opioids consumption, less postoperative nausea and vomiting compared with other analgesic treatment strategies, indicating that a postoperative PVB is a feasible and an effective method for an improved postoperative pain treatment after abdominal surgery. This study demonstrated that realtime ultra-sound PVB block is effective and safe technique. The study had demonstrated its superiority over standard medical therapy for postoperative pain control. The use of ultrasound for the placement of 
nerve blocks had proved its efficacy in clinical practice with more intense analgesia and fewer side effects.

\section{REFERENCES}

1. Alagol A (2012): Anesthetic management of abdominal surgery. In Abdominal Surgery. In Tech., 22:12-19.

2. Smith I, Kranke P, Murat I, Smith A, O'sullivan G, Søreide E and Spies C (2011): Perioperative fasting in adults and children: guidelines from the European Society of Anaesthesiology. European Journal of Anaesthesiology (EJA), 28(8): 556-569.

3. Joshi GP, Bonnet F, Shah R, Wilkinson RC, Camu F, Fischer B and Kehlet $H$ (2008): A systematic review of randomized trials evaluating regional techniques for postthoracotomy analgesia. Anesthesia \& Analgesia, 107(3): 1026-1040.

4. Eid HE (2009): Paravertebral block: An overview. Current Anaesthesia and Critical Care, 20(2): 65-70.

5. Klein SM, Bergh A, Steele SM, Georgiade GS and Greengrass RA (2010): Thoracic paravertebral block for breast surgery. Anesthesia \& Analgesia, 90(6): 1402-1405.

6. Agarwal A, Batra RK, Chhabra A, Subramaniam R and Misra MC (2012): The evaluation of efficacy and safety of paravertebral block for perioperative analgesia in patients undergoing laparoscopic cholecystectomy. Saudi Journal of Anaesthesia, 6(4): 344.

7. Fentie DY, Gebremedhn EG, Denu ZA and Gebreegzi AH (2017): Efficacy of single-injection unilateral thoracic paravertebral block for post open cholecystectomy pain relief: a prospective randomized study at Gondar University Hospital. Local and Regional Anesthesia, 10: 67-80.

8. Ben-David B, Swanson J, Nelson JB and Chelly JE (2007): Multimodal analgesia for radical prostatectomy provides better analgesia and shortens hospital stay. Journal of Clinical Anesthesia, 19(4): 264-268.

9. Naja Z, Ziade MF and Lönnqvist PA (2002): Bilateral paravertebral somatic nerve block for ventral hernia repair. European Journal of Anaesthesiology, 19(3): 197-202.
10. Jin LJ, Wen LY, Zhang YL, Li G, Sun P and Zhou X (2017): Thoracic paravertebral regional anesthesia for pain relief in patients with breast cancer surgery. Medicine, 96(39): 125-8.

11. Kehlet $H$ and Dahl JB (2003): Anaesthesia, surgery, and challenges in postoperative recovery. The Lancet, 362(9399): 1921-1928.

12. Matthews PJ and Govenden V (1989): Comparison of continuous paravertebral and extradural infusions of bupivacaine for pain relief after thoracotomy. British Journal of Anaesthesia, 62(2): 204-205. 